\title{
Synthesis of a Dye Containing Benzothiadiazole for Highly Efficient Dye-sensitized Solar Cells
}

\author{
Chao-Feng Du \\ Hubei Key Laboratory of Natural Products Research \\ and Development, College of Biological and \\ Pharmaceutical Sciences, China Three Gorges \\ University, Yichang, China. \\ duchaofeng1990110@163.com
}

\section{Lei Jiang}

State Key Laboratory of Molecular Reaction Dynamics, Dalian National Laboratory for Clean Energy, Dalian Institute of Chemical Physics Chinese Academy of Science, Dalian, China leijiang@dicp.ac.cn

\section{Nian-Yu Huang}

Hubei Key Laboratory of Natural Products Research and Development, College of Biological and Pharmaceutical Sciences, China Three Gorges University, Yichang, China. hny115@126.com (corresponding author)
Triarylamine compounds as a very good donor was used for dye-sensitized solar cells (DSSCs) has been widely applied. The different bridge and acceptor used will shows different power conversion efficiency. In this work, We synthesized two new dyes (D1 and D2) containing benzothiadiazole structure as dye-sensitized solar cells (DSSCs) with 3,4-(ethylenedioxy)thiophene bridged, a donor of triphenylamine and an acceptor of cyanoacrylic acid. D2 compared with D1 adds a phenyl ring. Compounds 6 and 7 were synthesized via Still coupling reactions. Comparing with dye-sensitized solar cells (DSSCs) based on dye D1, the dye-sensitized solar cells (DSSCs) based on D2 exhibited a significant enhancement of cell performances. A power conversion efficiency of $\mathbf{7 . 7 \%}$ was achieved for dye-sensitized solar cells (DSSCs) based on D2, which was much higher than that for dye-sensitized solar cells (DSSCs) based on D1 $(0.9 \%)$. D2 through introducing a $\pi$-conjugated spacer unit (phenyl ring) between the benzothiadiazole unit and the cyanoacetic acid group shows outstanding performance.

Keywords-component; benzothiadiazole; Triarylamine; Photovoltaic Performane; sensitizer

\section{Introduction}

Dye sensitized solar cells (DSSCs) are regarded as the environment-friendly and low-cost solar energy conversion device[1-4]. Generally, organic dyes are consisted of a donor- $\pi$-acceptor (D- $\pi$-A) type structure, of which $\mathrm{D}$ is an electron-donating unit, $\pi$ is a $\pi$-conjugated spacer unit, and A is an electron-accepting unit. The most common D- $\pi$-A type dyes were consisted of a

\author{
Lei Sun \\ State Key Laboratory of Molecular Reaction Dynamics, \\ Dalian National Laboratory for Clean Energy, Dalian \\ Institute of Chemical Physics Chinese Academy of \\ Science, Dalian, China \\ slei@dicp.ac.cn \\ Wei-Qiao Deng \\ State Key Laboratory of Molecular Reaction Dynamics, \\ Dalian National Laboratory for Clean Energy, Dalian \\ Institute of Chemical Physics Chinese Academy of \\ Science, Dalian, China \\ dengwq@dicp.ac.cn
}

triphenylamine group as donor, a cyanoacetic acid group as acceptor, and a thiophene (T) or its derivatives as $\pi$ spacer[5-9]. For example, the cell power conversion efficiency of DSSCs based on C219 can reach as high as $10.3 \%$ [10]. Here, we attempt to search new metal-free organic dyes for DSSCs. Two new D- $\pi$-A structure dyes D1 and D2 have been synthesized. Both of them are made up with triphenylamine as donor group and cyanoacetic acid as acceptor group. Dye D1 takes a benzothiadiazole (BTDA) group as the $\pi$-spacer and BTDA group is directly adjacent with cyanoacetic acid group. On the other hand, dye D2 adds a phenyl group between BTDA and cyanoacetic acid group. Both structures are shown in the following scheme.

\section{Experimental}

Compound 4

(4-[5-tributylstannyl-3,4ethylenedioxythiophene-2-yl]-N,N-bis(4-hexyloxyphenyl)aniline) and 5 (4-Bromo-7-(4-formylphenyl)-2,1,3-benzothiadiazole) was synthesized according to the literature procedures $[11,12]$. Compounds 6 (7-\{4-[N,N-Bis(4-hexyloxyphenyl)-4aminophenyl]-3,4-ethylenedioxythiophene-2-yl $\}$ benzo[1,2,5] thiadiazole-4-carbaldehyde) and 7 (4-\{7-\{4-[N,N-Bis(4hexylox-yphenyl)-4-aminophenyl]-3,4-ethylenedioxythiophene2-yl\}benzo[1,2,5]thiadiazole-4-yl \}ben-zaldehyde) were synthesized via Stillcoupling reactions. All reagents were purchased from Aladdin, TCI or Alfa Aesar, and used as received without further purification. The solvents were purified by distillation under a nitrogen atmosphere prior to use. $1 \mathrm{H}$ NMR and 13C NMR spectra were recorded on a BRUKER 
AVANCE $400 \mathrm{MHz}$ NMR instrument by using $\mathrm{CDCl} 3$ or DMSO-d6 as solvent using tetramethylsilane as an internal reference. MALDI-TOF was performed on an AB SCIEX instrument using CHCA as a matrix.
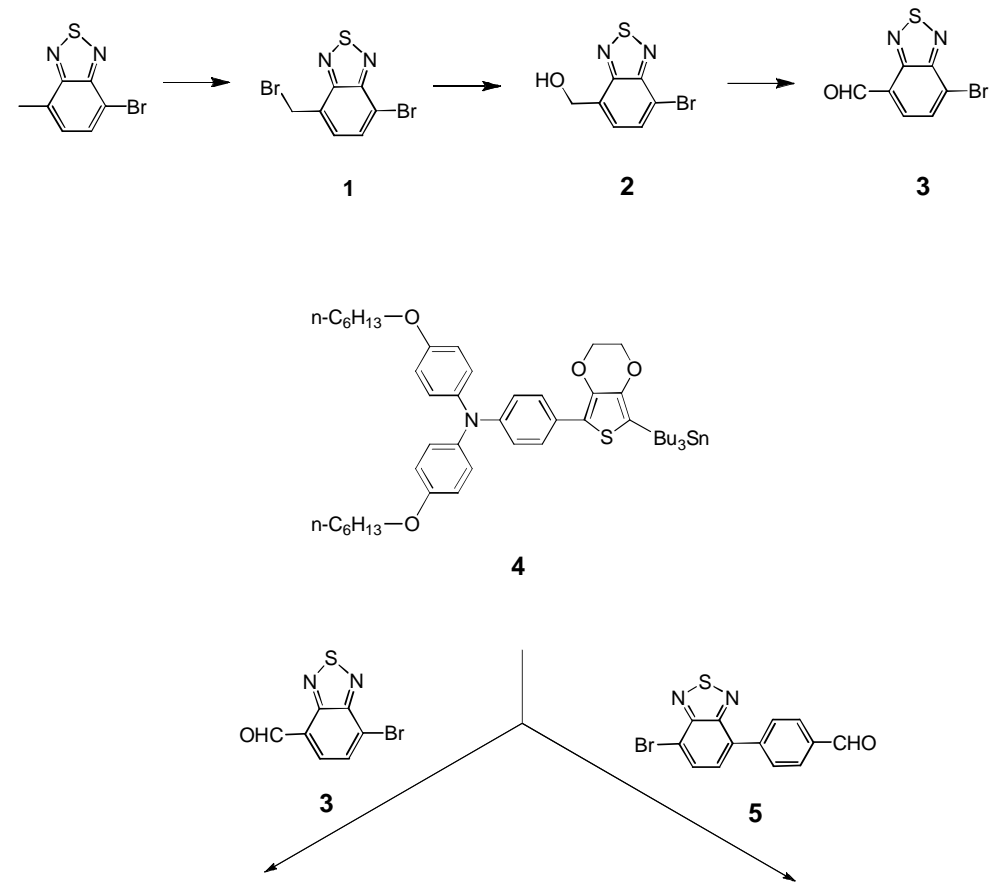

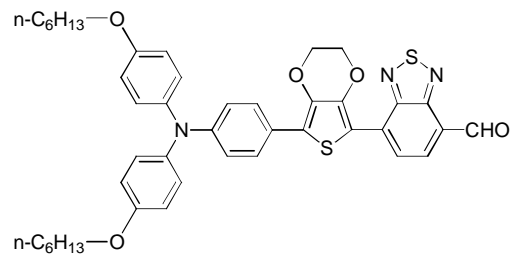

6

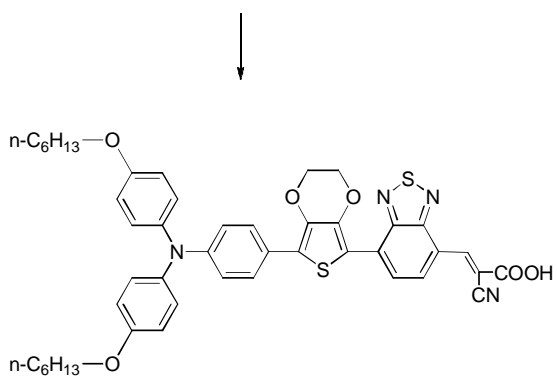

D1

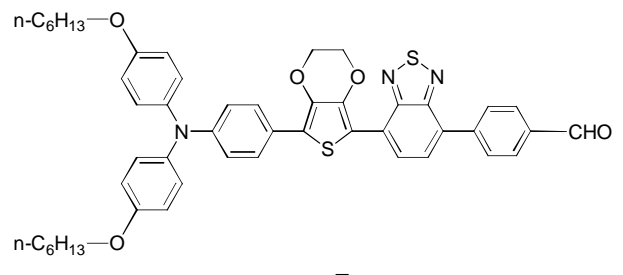

7
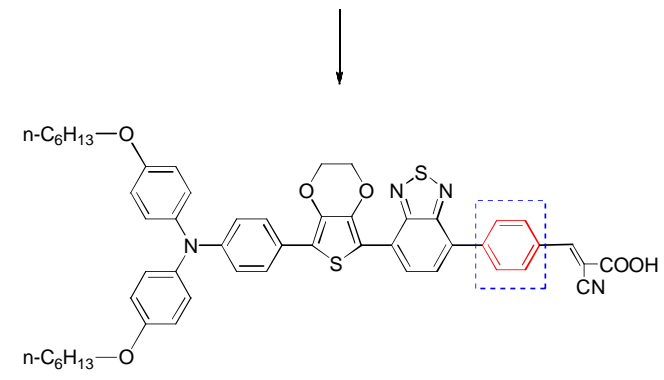

D2

Scheme 1. The synthetic routes for the dyes D1 and D2

\section{A. 4-Bromo-7-bromomethylbenzo-2,1,3-thiadiazole (1)}

4-Bromo-7-methylbenzo-2,1,3-thiadiazole (4.8 g, $21 \mathrm{mmol}$ ), benzoyl peroxide $(15 \mathrm{mg}), 33 \% \mathrm{HBr}$ in $\mathrm{AcOH}$ $(1 \mathrm{~mL})$ and NBS $(3.7 \mathrm{~g}, 21 \mathrm{mmol})$ were dissolved in $\mathrm{CCl}_{4}$ $(150 \mathrm{ml})$, the mixture was stirred at room temperature for 30 minutes, The reaction was quenched by addition of water and extracted with DCM. The combined organic extract was dried over anhydrous $\mathrm{MgSO} 4$ and filtered, The crude product was purified by column chromatography (hexane/ dichloromethane) gave compound 1 (5.45 g, 85\%). ${ }^{1} \mathrm{H}$ NMR $\left(400 \mathrm{MHz}, \mathrm{CDCl}_{3}\right) \delta$ : $7.83(\mathrm{~d}, \mathrm{~J}=7.6 \mathrm{~Hz}, 1 \mathrm{H}), 7.55(\mathrm{~d}, \mathrm{~J}=7.6 \mathrm{~Hz}, 1 \mathrm{H}), 4.94(\mathrm{~s}$, $2 \mathrm{H}) .{ }^{13} \mathrm{C}$ NMR $\left(400 \mathrm{MHz}, \mathrm{CDCl}_{3}\right) \delta: 153.5,152.9,131.9$, $130.3,129.8,114.8,27.7$.

B. 4-Bromo-7-hydroxymethylbenzo-2,1,3-thiadiazole (2)

The compound 1 (5 g, $16 \mathrm{mmol})$ was dissolved in a mixture of dioxane/water $(100 \mathrm{~mL}, 1: 1 \mathrm{v} / \mathrm{v}), \mathrm{K}_{2} \mathrm{CO}_{3}(6.72$ $\mathrm{g}, 48 \mathrm{mmol}$ ) was added to the mixture then refluxed for 1 $\mathrm{h}$, the solvents were removed in vacuo. The residue was acidified with $20 \%$ aqueous $\mathrm{HCl}$ and extracted with dichloromethane. The organic layer was dried over $\mathrm{MgSO}_{4}$, the solvent was removed in vacuo. The crude 
product was purified by column chromatography (hexane/ dichloromethane) gave compound $2(2.02 \mathrm{~g}, 51 \%) .{ }^{1} \mathrm{H}$ NMR (400 MHz, $\left.\mathrm{CDCl}_{3}\right) \delta: 7.84(\mathrm{~d}, \mathrm{~J}=7.2 \mathrm{~Hz}, 1 \mathrm{H})$, $7.49(\mathrm{~d}, \mathrm{~J}=7.2 \mathrm{~Hz}, 1 \mathrm{H}), 5.14(\mathrm{~d}, \mathrm{~J}=5.6 \mathrm{~Hz}, 2 \mathrm{H}), 2.58(\mathrm{t}, \mathrm{J}$ $=6.4 \mathrm{~Hz}, 1 \mathrm{H}) .{ }^{13} \mathrm{C} \mathrm{NMR}\left(400 \mathrm{MHz}, \mathrm{CDCl}_{3}\right) \delta: 153.5$, $153.1,133.2,132.0,126.9,113.2,61.8$.

\section{7-Bromobenzo[1,2,5]thiadiazole-4-carbaldehyde (3)}

The compound 2 (1.5 g, $6 \mathrm{mmol})$ was dissolved in chloroform, Manganese dioxide (2.09 g, $24 \mathrm{mmol}$ ) added to the mixture, the mixture was stirred at room temperature for $15 \mathrm{~h}$, the reaction mixture was filtered, the solvent was removed in vacuo. The crude product was purified by column chromatography (hexane/ dichloromethane) gave compound $3(1.28 \mathrm{~g}, 85 \%) .{ }^{1} \mathrm{H}$ $\operatorname{NMR}\left(400 \mathrm{MHz}, \mathrm{CDCl}_{3}\right) \delta: 10.78(\mathrm{~s}, 1 \mathrm{H}), 8.11(\mathrm{~d}, \mathrm{~J}=7.6$ $\mathrm{Hz}, 1 \mathrm{H}), 8.06(\mathrm{~d}, \mathrm{~J}=7.6 \mathrm{~Hz}, 1 \mathrm{H}) .{ }^{13} \mathrm{C}$ NMR $(400 \mathrm{MHz}$, $\left.\mathrm{CDCl}_{3}\right) \delta: 188.4,154.1,152.4,132.2,131.7,126.9,121.9$.

\section{7-\{4-[N,N-Bis(4-hexyloxyphenyl)-4-aminophenyl]-}

3,4-ethylenedioxythiophene-2-

yl\}benzo[1,2,5]thiadiazole-4-carbaldehyde (6)

The compound 4 (300 mg, $0.34 \mathrm{mmol}$ ), the compound 3 (109 $\mathrm{mg}$, $0.45 \quad \mathrm{mmol}) \quad$ and bis(triphenylphosphine)palladium(II) chloride $(60 \mathrm{mg})$ were dissolved under argon in dry toluene $(50 \mathrm{~mL})$, the mixture was heated at $100^{\circ} \mathrm{C}$ under argon atmosphere for $12 \mathrm{~h}$. After cooling to room temperature, the reaction mixture was dissolved in dichloromethane $(3 \times 50 \mathrm{~mL})$, washed with water $(2 \times 20 \mathrm{~mL})$ and dried over $\mathrm{MgSO}_{4}$, the solvent was removed in vacuo. The crude product was purified by column chromatography (hexane/dichloromethane) gave compound 6 (185 mg, $73 \%) .{ }^{1} \mathrm{H}$ NMR $\left(400 \mathrm{MHz}, \mathrm{CDCl}_{3}\right) \delta: 10.54(\mathrm{~s}, 1 \mathrm{H}), 8.58$ $(\mathrm{d}, \mathrm{J}=8.0 \mathrm{~Hz}, 1 \mathrm{H}), 8.30(\mathrm{~d}, \mathrm{~J}=8.0 \mathrm{~Hz}, 1 \mathrm{H}), 7.63(\mathrm{~d}, \mathrm{~J}=$ $8.8 \mathrm{~Hz}, 2 \mathrm{H}$ ), 7.05 (d, J = 8.8 Hz, 4H ), 6.93 (d, J = 8.8 Hz, $4 \mathrm{H}), 6.83(\mathrm{~d}, \mathrm{~J}=8.8 \mathrm{~Hz}, 2 \mathrm{H}), 4.56(\mathrm{~m}, 2 \mathrm{H}), 4.44(\mathrm{~m}$, $2 \mathrm{H}), 3.96(\mathrm{t}, \mathrm{J}=6.4 \mathrm{~Hz}, 4 \mathrm{H}), 1.74(\mathrm{~m}, 4 \mathrm{H}), 1.44(\mathrm{~m}$, $4 \mathrm{H}), 1.33(\mathrm{~m}, 8 \mathrm{H}), 0.90(\mathrm{t}, \mathrm{J}=7.2 \mathrm{~Hz}, 6 \mathrm{H}) .{ }^{13} \mathrm{C} \mathrm{NMR}$ $\left(100 \mathrm{MHz}, \mathrm{CDCl}_{3}\right) \delta: 188.4,155.8,153.6,152.3,148.5$, $143.8,137.2,132.5,127.4,123.5,119.9,115.4,68.3,65.2$, $64.3,31.9,31.6,29.7,29.3,25.8,22.6,14.0$. MALDITOF $(\mathrm{m} / \mathrm{z}): 747.7\left[\mathrm{M}^{+}\right]$.

\section{E. 4-\{7-\{4-[N,N-Bis(4-hexyloxyphenyl)-4- \\ aminophenyl]-3,4-ethylenedioxythiophene-2- \\ yl\}benzo[1,2,5]thiadiazole-4-yl\} benzaldehyde (7)}

The compound 4 (300 mg, $0.34 \mathrm{mmol})$, the compound 5 (143 $\mathrm{mg}, \quad 0.45 \quad \mathrm{mmol}) \quad$ and bis(triphenylphosphine)palladium(II) chloride (60 $\mathrm{mg})$ were dissolved under argon in dry toluene $(50 \mathrm{~mL})$, the mixture was heated at $100^{\circ} \mathrm{C}$ under argon atmosphere for $12 \mathrm{~h}$. After cooling to room temperature, the reaction mixture was dissolved in dichloromethane $(3 \times 50 \mathrm{~mL})$, washed with water $(2 \times 20 \mathrm{~mL})$ and dried over $\mathrm{MgSO}_{4}$, the solvent was removed in vacuo. The crude product was purified by column chromatography (hexane/dichloromethane) gave compound 7 (87 $\mathrm{mg}$, 31\%). ${ }^{1} \mathrm{H}$ NMR $\left(400 \mathrm{MHz}, \mathrm{CDCl}_{3}\right.$ ) $\delta: 10.09$ (s, 1H ), 8.47 $(\mathrm{d}, \mathrm{J}=8.0 \mathrm{~Hz}, 1 \mathrm{H}), 8.17(\mathrm{~d}, \mathrm{~J}=8.0 \mathrm{~Hz}, 2 \mathrm{H}), 8.03(\mathrm{~d}, \mathrm{~J}=$ $8.0 \mathrm{~Hz}, 2 \mathrm{H}), 7.81(\mathrm{~d}, \mathrm{~J}=8.0 \mathrm{~Hz}, 1 \mathrm{H}), 7.68(\mathrm{~d}, \mathrm{~J}=8.8 \mathrm{~Hz}$, $2 \mathrm{H}), 7.08(\mathrm{~d}, \mathrm{~J}=9.2 \mathrm{~Hz}, 4 \mathrm{H}), 6.96(\mathrm{~d}, \mathrm{~J}=8.4 \mathrm{~Hz}, 2 \mathrm{H})$, $6.84(\mathrm{~d}, \mathrm{~J}=9.2 \mathrm{~Hz}, 4 \mathrm{H}), 4.45(\mathrm{~m}, 2 \mathrm{H}), 4.39(\mathrm{~m}, 2 \mathrm{H})$, $3.96(\mathrm{t}, \mathrm{J}=6.4 \mathrm{~Hz}, 4 \mathrm{H}), 1.82(\mathrm{~m}, 4 \mathrm{H}), 1.49(\mathrm{~m}, 4 \mathrm{H})$, $1.37(\mathrm{~m}, 8 \mathrm{H}), 0.94(\mathrm{t}, \mathrm{J}=7.2 \mathrm{~Hz}, 6 \mathrm{H}) .{ }^{13} \mathrm{C} \mathrm{NMR}(100$ $\left.\mathrm{MHz}, \mathrm{CDCl}_{3}\right) \delta: 191.8,155.6,153.4,152.7,147.9,143.5$, $141.7,140.5,137.1,135.5,129.9,129.6,129.3,128.9$, $127.2,126.7,125.4,124.7,121.7,120.3,115.3,110.1$, $68.3,64.9,64.4,31.6,29.3,25.8,22.6,14.0$. MALDITOF (m/z): $823.7\left[\mathrm{M}^{+}\right]$

\section{F. 2-Cyano-3-\{7-\{4-[N,N-Bis(4-hexyloxyphenyl)-4- aminophenyl]-3,4-ethylenedioxythiophene-2- yl\}benzo[1,2,5]thiadiazole-4-yl\}acrylic acid (D1):}

Compound 6 (150 mg, $0.21 \mathrm{mmol})$, ammonium acetate $(16 \mathrm{mg}, 0.21 \mathrm{mmol})$ and cyanoacetic acid $(179 \mathrm{mg}, 2.2$ $\mathrm{mmol})$ were dissolved in a mixture of $\mathrm{DCM} / \mathrm{MeCN}(60$ $\mathrm{mL}, 2: 1 \mathrm{v} / \mathrm{v})$. The mixture was refluxed under argon atmosphere for $10 \mathrm{~h}$. After cooling to room temperature, the reaction mixture was dissolved in dichloromethane (3 $\times 50 \mathrm{~mL})$, washed with water $(2 \times 20 \mathrm{~mL})$ and dried over $\mathrm{MgSO}_{4}$. The solvent was removed in vacuo. The crude product was purified by column chromatography (dichloromethane/methanol) to obtained dye D1 as a dark blue solid (111 mg, 65\%). ${ }^{1} \mathrm{H}$ NMR (400 MHz, $\left.\mathrm{CDCl}_{3}\right) \delta$ : $9.26(\mathrm{~s}, 1 \mathrm{H}), 8.92(\mathrm{~d}, \mathrm{~J}=7.6 \mathrm{~Hz}, 1 \mathrm{H}), 8.59(\mathrm{~d}, \mathrm{~J}=7.6 \mathrm{~Hz}$, $1 \mathrm{H}), 7.69(\mathrm{~d}, \mathrm{~J}=8.4 \mathrm{~Hz}, 2 \mathrm{H}), 7.08(\mathrm{~d}, \mathrm{~J}=8.4 \mathrm{~Hz}, 4 \mathrm{H})$, $6.95(\mathrm{~d}, \mathrm{~J}=8.4 \mathrm{~Hz}, 2 \mathrm{H}), 6.85(\mathrm{~d}, \mathrm{~J}=8.8 \mathrm{~Hz}, 4 \mathrm{H}), 4.52(\mathrm{~m}$, $2 \mathrm{H}), 4.42(\mathrm{~m}, 2 \mathrm{H}), 3.96(\mathrm{t}, \mathrm{J}=6.0 \mathrm{~Hz}, 4 \mathrm{H}), 1.82(\mathrm{~m}$, $4 \mathrm{H}), 1.51(\mathrm{~m}, 4 \mathrm{H}), 1.37(\mathrm{~m}, 8 \mathrm{H}), 0.94(\mathrm{~m}, 6 \mathrm{H}) .{ }^{13} \mathrm{C}$ NMR (100 MHz, DMSO-d $\left.{ }_{6}\right) \delta: 155.4,153.9,150.7,147.7$, $142.8,139.8,139.5,137.3,128.1,127.2,126.8,126.7$, $124.4,123.8,122.7,121.0,119.1,115.5,108.9,67.6,65.1$, $64.2,30.9,28.7,25.2,22.0,13.8$. MALDI-TOF $(\mathrm{m} / \mathrm{z})$ : $814.6\left[\mathrm{M}^{+}\right]$

\section{G. 3-\{4-\{7-\{4-[N,N-Bis(4-hexyloxyphenyl)-4- aminophenyl]-3,4-ethylenedioxythiophene-2- yl\}benzo[1,2,5]thiadiazole-4-yl\} phenyl \}-2- cyanoacrylic acid (D2):}

Compound 7 (85 mg, $0.10 \mathrm{mmol})$, ammonium acetate $(8$ $\mathrm{mg}, 0.1 \mathrm{mmol})$ and cyanoacetic acid $(80 \mathrm{mg}, 1.0 \mathrm{mmol})$ were dissolved in a mixture of $\mathrm{DCM} / \mathrm{MeCN}(60 \mathrm{~mL}, 2: 1$ $\mathrm{v} / \mathrm{v})$. The mixture was refluxed under argon atmosphere for $24 \mathrm{~h}$. After cooling to room temperature, the reaction mixture was dissolved in dichloromethane $(3 \times 50 \mathrm{~mL})$, washed with water $(2 \times 20 \mathrm{~mL})$ and dried over $\mathrm{MgSO}_{4}$. The solvent was removed in vacuo. The crude product was purified by column chromatography (dichloromethane/methanol) to was obtained dye $\mathbf{D 2}$ as a dark blue solid (41 mg, 45\%). ${ }^{1} \mathrm{H}$ NMR (400 MHz, DMSO $\left.\mathrm{d}_{6}\right) \delta: 8.51(\mathrm{~d}, \mathrm{~J}=8.0 \mathrm{~Hz}, 1 \mathrm{H}), 8.18(\mathrm{~d}, \mathrm{~J}=8.4 \mathrm{~Hz}$, $2 \mathrm{H}), 8.05(\mathrm{~m}, 4 \mathrm{H}), 7.61(\mathrm{~d}, \mathrm{~J}=8.8 \mathrm{~Hz}, 2 \mathrm{H}), 7.03(\mathrm{~d}, \mathrm{~J}=$ $8.8 \mathrm{~Hz}, 4 \mathrm{H}), 6.92(\mathrm{~d}, \mathrm{~J}=8.8 \mathrm{~Hz}, 4 \mathrm{H}), 6.84(\mathrm{~d}, \mathrm{~J}=8.8 \mathrm{~Hz}$, $2 \mathrm{H}), 4.52(\mathrm{~m}, 2 \mathrm{H}), 4.42(\mathrm{~m}, 2 \mathrm{H}), 3.95(\mathrm{t}, \mathrm{J}=6.4 \mathrm{~Hz}$, $4 \mathrm{H}), 1.74(\mathrm{~m}, 4 \mathrm{H}), 1.45(\mathrm{~m}, 4 \mathrm{H}), 1.33(\mathrm{~m}, 8 \mathrm{H}), 0.90(\mathrm{~m}$, $6 \mathrm{H}) \cdot{ }^{13} \mathrm{C}$ NMR $\left(100 \mathrm{MHz}, \mathrm{DMSO}-\mathrm{d}_{6}\right) \delta: 155.8,153.3$, $152.3,147.9,142.4,140.1,137.8,129.9,129.7,127.3$, $127.2,125.8,124.6,120.2,119.9,115.9,99.9,68.1,31.5$, 29.2, 25.7, 22.5, 14.4. MALDI-TOF (m/z): $890.7\left[\mathrm{M}^{+}\right]$.

\section{Results and discussion}

Fig .1 indicated the current-voltage $(J-V)$ characteristics of DSSCs sensitized by D1 and D2 dyes, employing a $\mathrm{I}^{-} / \mathrm{I}_{3}{ }^{-}$electrolyte measured under one sun solar illumination conditions (AM $1.5 \mathrm{G}, 100 \mathrm{~mW} \mathrm{~cm}^{-2}$ ). 


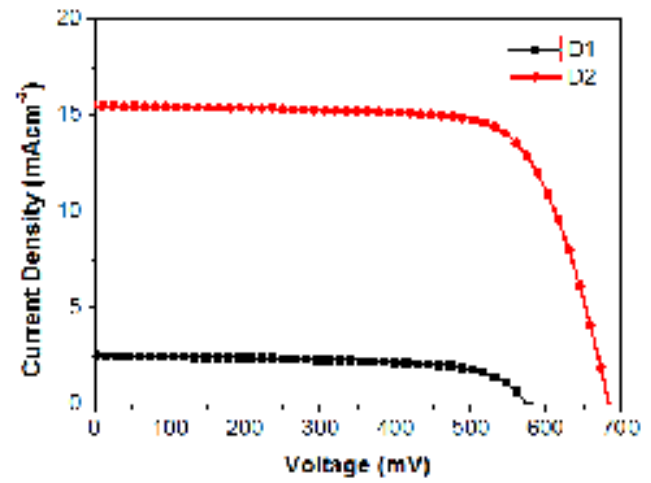

Figure 1. $J-V$ curves of DSSCs sensitized with D1 and D2.

Fig .2 showed the incident photon-to-current conversion efficiency (IPCE) spectra of DSSCs sensitized with D1 and D2 dyes. The IPCE spectra of cells sensitized with D1 and D2 dyes show a wide spectral coverage region. The max IPCE at $550 \mathrm{~nm}$ is only $14 \%$ for $\mathrm{D} 1$ dye and $77 \%$ for $\mathrm{D} 2$ dye.

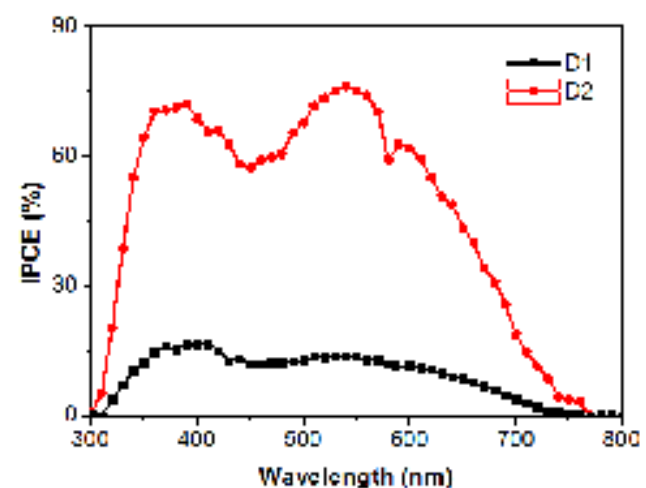

Figure 2. IPCE spectra for DSSCs sensitized with D1 and D2.

The photovoltaic parameters of DSSCs were summarizedin Table I. The DSSCs sensitized by D2 dye provided a power conversion efficiencyof $7.7 \%$ with high short-circuit photocurrent density $\left(J_{s c}\right) \quad 15.51 \mathrm{~mA} / \mathrm{cm}^{2}$, high open-circuit photovoltage $\left(V_{o c}\right) 683 \mathrm{mV}$ and high fill factor $(F F) 72.5 \%$. Under the same conditions, the cellssensitized with D1 dye showeda poor performancedue to lower $J_{s c}$ and $V_{o c}$ values $\left(\eta=1.0 \%, J_{s c}\right.$ $=2.58 \mathrm{~mA} / \mathrm{cm}^{2}, V_{o c}=574 \mathrm{mV}, F F=64.5 \%$ ).

TABLE I. PHOTOVOLTAIC PARAMETERS OF DSSCS SENSITIZED WITH D1 AND D2.

\begin{tabular}{ccccc}
\hline Dye & $\begin{array}{c}\text { Short-circuit } \\
\text { current density } \\
(\mathbf{m A c m})^{-2}\end{array}$ & $\begin{array}{c}\text { Open- } \\
\text { circuit } \\
\text { voltage } \\
(\mathbf{m V})\end{array}$ & $\begin{array}{c}\text { Fill } \\
\text { factor } \\
(\%)\end{array}$ & $\begin{array}{c}\text { Light-to- } \\
\text { electrical energy } \\
\text { conversion } \\
\text { effieiene }(\%)\end{array}$ \\
\hline D1 & 2.58 & 574 & 64.5 & 1.0 \\
\hline D2 & 15.51 & 683 & 72.5 & 7.7 \\
\hline
\end{tabular}

\section{Conclusions}

In conclusion, we synthesized two new dyes (D1 and D2) through introducing a $\pi$-conjugated spacer unit (phenyl ring) between the benzothiadiazole unit and the cyanoacetic acid group, which exhibited a great enhancement on the photovoltaic performance. The cell power conversion efficiency of DSSCs based on D2 can reach as high as $7.7 \%$, which was superior to that of DSSCs based on D1 (1.0\%).

\section{Acknowledgements}

We gratefully acknowledge the financial support from the National Natural Science Foundation of China (Nos. 9133316, 21403211).

\section{References}

[1] A. Hagfeldt, G. Boschloo, L. Sun, L. Kloo and H. Pettersson, "Dyesensitized solar cells," Chemical Reviews, vol. 110, Sept. 2010, pp. 6595-6663, doi: 10.1021/cr900356p

[2] A. Hagfeldt and M. Grätzel, "Light-induced redox reactions in nanocrystalline systems," Chemical Reviews, vol. 95, Jan. 1995, pp. 49-68, doi: 10.1021/cr00033a003.

[3] B. O'Regan and M. Grätzel, "A low-cost, high-efficiency solar cell based on dye sensitized colloidal $\mathrm{TiO}_{2}$ films," Nature, vol. 353, 1991, pp. 737-740, doi: 10.1038/353737a0.

[4] M. T. Spitler and B. A. Parkinson, "Dye sensitization of single crystal semiconductor electrodes," Accounts of Chemical Research, vol. 42, Nov. 2009, pp. 2017-2029, doi: 10.1021/ar900232m.

[5] H. H. Chou, Y. C. Chen, H. J. Huang, T. H. Lee, J. T. Lin, C. Tsai and K. Chen, "High-performance dye-sensitized solar cells based on 5,6-bis-hexyloxy-benzo[2,1,3] thiadiazole," Journal of Materials Chemistry, vol. 22, Jan. 2012, pp. 10929-10938, doi: $10.1039 / \mathrm{c} 2 \mathrm{jm} 30427 \mathrm{a}$.

[6] G. Li, K. J. Jiang, Y. F. Li, S. L. Li and L. M. Yang, "Efficient structural modification of triphenylamine-based organic dyes for dye-sensitized solar cells," Journal of Physical Chemistry C, vol. 112, Jul. 2008, pp. 11591-11599, doi: 10.1021/jp802436v.

[7] W. H. Liu, I. C. Wu, C. H. Lai, P. T. Chou, Y. T. Li, C. L. Chen, Y. Y. Hsu and Y. Chi, "Simple organic molecules bearing a 3,4ethylenedioxythiophene linker for efficient dye-sensitized solar cells," Chemical Communications, vol. 41, May 2008, pp. 51525154, doi: 10.1039/b808535h.

[8] C. J. Qin, A. Islam and L. Y. Han, “ Incorporating a stable fluorenone unit into D-A-pi-A organic dyes for dye-sensitized solar cells," Journal of Materials Chemistry A, vol. 22, May 2012, pp. 19236-19243, doi: 10.1039/c2jm33105e.

[9] R. Z. Li, X. J. Lv, D. Shi, D. F. Zhou, Y. M. Cheng, G. L. Zhang and P. Wang, "Dye-sensitized solar cells based on organic sensitizers with different conjugated linkers: furan, bifuran, thiophene, bithiophene, selenophene, and biselenophene," Journal of Physical Chemistry C, vol. 113, Apr. 2009, pp. 7469-7479, doi: 10.1021/jp900972v.

[10] W. Zeng, Y. Cao, Y. Bai, Y. Wang, Y. Shi, M. Zhang, "efficient dyesensitized solar cells with an organic photosensitizer featuring orderly conjugated ethylenedioxythiophene and dithienosilole blocks,"chemistry of materials, vol. 22, Jan. 2010, pp.1915-1925, doi: $10.1021 / \mathrm{cm} 9036988$.

[11] X. L. Hao , M. Liang, X. B. Cheng, X. Q. Pian, Z. Sun, S. Xue, "Organic Dyes Incorporating the Benzo[1,2-b:4,5-b']dithiophene Moiety for Efficient Dye-Sensitized Solar Cells,"Organic Letters, vol. 13, Sept. 2011, pp. 5424-5427, doi: 10.1021/ol201858b.

[12] A. S. D. Sandanayaka, Y. Taguri, Y. Araki, T. Ishi-i, S. Mataka, O. Ito, "Photoinduced Charge Separation and Charge Recombination in the [60]Fullerene-Diphenylbenzothiadiazole-Triphenylamine Triad: Role of Diphenylbenzothiadiazole as Bridge,"Journal of Physical Chemistry B, vol. 109, Nov. 2005, pp. 22502-22512, doi: $10.1021 /$ jp053809c. 